\title{
Electromagnetic nature of the nuclear forces and toroid structure of the deuteron and triton
}

\author{
Kiril Kolikov*, Dragiya Ivanov, Georgi Krastev

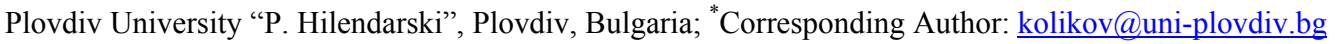

Received 15 December 2011; revised 10 January 2012; accepted 19 January 2012

\begin{abstract}
In this paper we model in a new way the nuclei of deuterium and tritium. We consider the nucleons as toroids that rotate at a constant angular velocity around a line perpendicular to their rotation plane and passing through the center of mass of the nuclei. Based on exact analytical formulas obtained by us for the electrostatic interaction between two spheres with arbitrary radii and charges, we obtain that the known binding energy of the deuteron and triton has an electromagnetic nature. We also obtain through these formulas the force of interaction inside these nuclei. Besides that, within the framework of the classical model we use, we calculate the volumes and mass densities of the nucleons. Throughout all that we use the experimentally obtained results for the radii and masses of the nucleons and nuclei under study. Through our toroid model we confirm the main experimental results obtained for the deuteron and triton not only for the binding energy but also for the magnetic moments, spins and stability.
\end{abstract}

Keywords: Deuteron; Triton; Strong Interactions; Binding Energy; Electrostatic Interactions

\section{INTRODUCTION}

In order for a complete theory of the atomic nucleus to be created, that theory has to be able to explain the structure of the nucleus and the available experimental data about its behavior [1-4]. The main difficulty here is the incomplete knowledge of the forces of interaction between nucleons inside the nuclei and their models.

In the field of elementary particles there are two leading models: standard and helicon.

The standard model [5-7] presents elementary particles as quantum objects that can be both wave and particle. As particles they have mass, charge, spin, magnetic moment, quadrupole moment. The elementary particles mainly are modelling with spheres.

The helicon model [8-11], or ring model is less wellknown. According to that model the particles have ring spiral structure of charged filaments, one or more. The filaments are superconductors coiled around an imaginary ring. The helicon model is consistent with all the widely accepted and experimentally verified properties of elementary particles. This model implies the assumption that all known types of interactions (strong, electroweak, gravitational) should be electromagnetic in nature.

In [12] we introduce a toroid model of the nucleons that is to a certain degree in contradiction with the standard model, but it is in full agreement with the helicon model.

We consider the nucleons as tori, rotating with a constant angular velocity around an axis $z$, passing through their mass (geometrical) centre $O$ and perpendicular to the plane of rotation of their central circle. From quantum mechanical point of view the nucleon is not a localized object in the three-dimensional physical space and therefore it cannot be consider a sphere or a torus [13]. We feel such a model is appropriate in the formal-heuristic sense of Niels Bohr. It is similar to the old quantum theory model of the electron in the hydrogen atom for which Bohr obtains good results for the description of its spectrums. Modern quantum theory confirms Bohr's results based on other concepts.

Formal approaches are widely used in physics. In classical mechanics for example a variety of formulations have been proposed by Lagrange, Hamilton and others. The best example in that sense is quantum mechanics, where one introduces the so-called wave function which allows for the theoretical derivation of a number of physical properties confirmed extremely well by experiment.

We consider nucleons within nuclei to be space dimensional objects - tori, within which the electrical charges can be redistributed. This assumption does not contradict the quark model. The latter enables us to determine the electrostatic interaction between them. Based on this model, we obtained that the strong interactions are electro- 
magnetic in nature. To achieve this we used exact analytical formulas first obtained by us about the electrostatic interaction between two charged conducting spheres with arbitrary charges and radii [14].

In this paper we apply this method in order to obtain the electrostatic interaction between nucleons in the nuclei of deuterium and tritium.

As noted by Feynman [15], at distances under $10^{-15} \mathrm{~m}$ either Coulomb's law is not in force or the electrons and protons are not point charges. We consider the proton-neutron couples at distances under $10^{-15} \mathrm{~m}$. Thus in this paper we determine that the known binding energy between the nucleons in the deuteron and triton is obtained through electromagnetic interactions! We also explain the other basic experimental data-spin, magnetic moment, stability of the nuclei of deuterium and tritium. Doing that, we use the experimentally obtained values of the radii and masses of the nucleons and the nuclei.

We obtain the volumes and mass densities of the nucleons; we also obtain the force of interaction within the nuclei under consideration-results obtained for the first time in nuclear physics.

\section{METHOD FOR FINDING OUT ELECTROSTATIC INTERACTION BETWEEN TWO CHARGED CONDUSTIVE SPHERES}

We will represent the part of the method presented by us in [10], necessary for performing the calculations for the nuclei of deuterium and tritium.

Let $S_{1}$ and $S_{2}$ be two isolated charged conductive spheres, with charges $Q_{1}, Q_{2}$ and radii $r_{1}, r_{2}$ respectively. Let's denote with $R$ the distance between their centers $O_{1}$, $\mathrm{O}_{2}$ in an inertial system $J$. Since charges $Q_{1}$ and $Q_{2}$ are evenly distributed on the surfaces of $S_{1}$ and $S_{2}$, it is assumed that before the interaction between the spheres they are concentrated in the centers $O_{1}$ and $O_{2}$ respectively.

As a result of the electrostatic interaction between $S_{1}$ and $S_{2}$, on their surfaces appear induced charges $\tilde{Q}_{1}$ and $\tilde{Q}_{2}$, which are interrelated. Formally, we can consider that these charges are located on line segment $\mathrm{O}_{1} \mathrm{O}_{2}$. On the surfaces of $S_{1}$ and $S_{2}$ appear uniformly distributed charges $\bar{Q}_{1}$ and $\bar{Q}_{2}$, we can assume that they are concentrated in their canters $\mathrm{O}_{1}$ and $\mathrm{O}_{2}$.

From the law for preservation of electric charges are in power the equations:

$$
O_{1} \bar{Q}_{1}=Q_{1}-\tilde{Q}_{1} \text { and } \bar{Q}_{2}=Q_{2}-\tilde{Q}_{2}
$$

We will determine the charges $\tilde{Q}_{1}, \tilde{Q}_{2}$ and hence the charges $\bar{Q}_{1}, \bar{Q}_{2}$. Let as a consequence of $Q_{1}$ be generated image charges $Q_{1, j}(j=1,2,3, \cdots)$. Because each charge $Q_{1, j}$ generates $Q_{1, j+1}$, the charges with an odd index $Q_{1,2 m-1}(m=1,2,3, \cdots)$ are located in the sphere $S_{2}$, and charges with an even index $Q_{1,2 m}$-in the sphere $S_{1}$. Similarly are determined the image charges $Q_{2, j} \quad(j=1,2,3, \cdots)$, arising as a consequence from charge $Q_{2}$. The charges with an odd index $Q_{2,2 m-1}(m=1,2,3$ $\cdots)$ are located in the sphere $S_{1}$, and charges with an even index $Q_{2,2 m}$-in the sphere $S_{2}$.

Let's denote $\delta_{1}=r_{1} / R$ and $\delta_{2}=r_{2} / R$. We introduce for $j=1,2,3, \cdots$ the following denotations:

$$
\begin{aligned}
& A_{1, j}=1+\sum_{k=1}^{j}(-1)^{k} \sum_{s=0}^{k}\left(\begin{array}{c}
j-1-s \\
k-s
\end{array}\right)\left(\begin{array}{c}
j-k+s \\
s
\end{array}\right) \delta_{1}^{2(k-s)} \delta_{2}^{2 s} \\
& A_{2, j}=1+\sum_{k=1}^{j}(-1)^{k} \sum_{s=0}^{k}\left(\begin{array}{c}
j-1-s \\
k-s
\end{array}\right)\left(\begin{array}{c}
j-k+s \\
s
\end{array}\right) \delta_{1}^{2 s} \delta_{2}^{2(k-s)} \\
& B_{1, j}=1+\sum_{k=1}^{j}(-1)^{k} \sum_{s=0}^{k}\left(\begin{array}{c}
j-s \\
k-s
\end{array}\right)\left(\begin{array}{c}
j-k+s \\
s
\end{array}\right) \delta_{1}^{2(k-s)} \delta_{2}^{2 s} \\
& B_{2, j}=1+\sum_{k=1}^{j}(-1)^{k} \sum_{s=0}^{k}\left(\begin{array}{c}
j-s \\
k-s
\end{array}\right)\left(\begin{array}{c}
j-k+s \\
s
\end{array}\right) \delta_{1}^{2 s} \delta_{2}^{2(k-s)}
\end{aligned}
$$

If $d_{i, j}(i=1,2 ; j=1,2,3, \cdots)$ are the distances of imagecharges $Q_{i, j}$, respectively to the centers of the spheres $O_{i}$, in [14] we obtain that:

$$
\begin{aligned}
& d_{1,2 m-1}=\delta_{2}^{2} R \frac{A_{1, m-1}}{B_{1, m-1}}, d_{1,2 m}=\delta_{1}^{2} R \frac{B_{1, m-1}}{A_{1, m}} \\
& d_{2,2 m-1}=\delta_{1}^{2} R \frac{A_{2, m-1}}{B_{2, m-1}}, d_{2,2 m}=\delta_{2}^{2} R \frac{B_{2, m-1}}{A_{2, m}}
\end{aligned}
$$

We find also that:

$$
\begin{aligned}
& Q_{1,2 m-1}=-\frac{\delta_{1}^{m-1} \delta_{2}^{m}}{B_{1, m-1}} \overline{Q_{1}}, Q_{1,2 m}=\frac{\delta_{1}^{m} \delta_{2}^{m}}{A_{1, m}} \overline{Q_{1}} \\
& Q_{2,2 m-1}=-\frac{\delta_{1}^{m} \delta_{2}^{m-1}}{B_{2, m-1}} \bar{Q}_{2}, Q_{2,2 m}=\frac{\delta_{1}^{m} \delta_{2}^{m}}{A_{2, m}} \bar{Q}_{2}
\end{aligned}
$$

Lets

$$
\begin{gathered}
X_{1}=\sum_{m=1}^{\infty} \frac{\delta_{1}^{m} \delta_{2}^{m}}{A_{1, m}}, X_{2}=\sum_{m=1}^{\infty} \frac{\delta_{1}^{m} \delta_{2}^{m}}{A_{2, m}} \\
Y_{1}=\sum_{m=1}^{\infty} \frac{\delta_{1}^{m-1} \delta_{2}^{m}}{B_{1, m-1}}, Y_{2}=\sum_{m=1}^{\infty} \frac{\delta_{1}^{m} \delta_{2}^{m-1}}{B_{2, m-1}}
\end{gathered}
$$

where $\delta_{i}^{0}=1$ at $\delta_{i}=0 \quad(i=1,2)$.

Since charges $\tilde{Q}_{1}$ and $\tilde{Q}_{2}$ are sums of all image charges, located respectively in the spheres $S_{1}$ and $S_{2}$, then

$$
\tilde{Q}_{1}=\sum_{m=1}^{\infty} Q_{1,2 m}+\sum_{m=1}^{\infty} Q_{2,2 m-1}
$$

and

$$
\tilde{Q}_{2}=\sum_{m=1}^{\infty} Q_{1,2 m-1}+\sum_{m=1}^{\infty} Q_{2,2 m}
$$

From here and from (4) and (5) it follows that 


$$
\tilde{Q}_{1}=\bar{Q}_{1} X_{1}-\bar{Q}_{2} Y_{2} \text { and } \tilde{Q}_{2}=-\bar{Q}_{1} Y_{1}+\bar{Q}_{2} X_{2}
$$

Then, substituting these equations in (1), we get:

$$
\begin{aligned}
& \bar{Q}_{1}=\frac{Q_{1}\left(1+X_{2}\right)+Q_{2} Y_{2}}{\left(1+X_{1}\right)\left(1+X_{2}\right)-Y_{1} Y_{2}} \\
& \bar{Q}_{2}=\frac{Q_{2}\left(1+X_{1}\right)+Q_{1} Y_{1}}{\left(1+X_{1}\right)\left(1+X_{2}\right)-Y_{1} Y_{2}}
\end{aligned}
$$

Lets denote the charges from formulas (4) and (6), which are located in the sphere $S_{1}$ as $Q_{j}^{\prime}$, and those located in the sphere $S_{2}$ as $Q_{j}^{\prime \prime}(j=0,1,2, \cdots)$. Thus $Q_{1,0}=\bar{Q}_{1}=Q_{0}^{\prime}$ and $Q_{2,0}=\bar{Q}_{2}=Q_{0}^{\prime \prime}$, and for $m=1,2,3, \cdots$, $Q_{2,2 m-1}=Q_{2 m-1}^{\prime} \quad, \quad Q_{1,2 m}=Q_{2 m}^{\prime} \quad$ and $Q_{1,2 m-1}=Q_{2 m-1}^{\prime \prime}$, $Q_{2,2 m}=Q_{2 m}^{\prime \prime}$. Their corresponding distances to the centers of the spheres, where they are situated, we denote with $d_{j}^{\prime}$ and $d_{j}^{\prime \prime}(j=0,1,2, \cdots)$, where $d_{0}^{\prime}=d_{0}^{\prime \prime}=0$.

If $\delta_{j}^{\prime}=d_{j}^{\prime} / R$, and $\delta_{j}^{\prime \prime}=d_{j}^{\prime \prime} / R$, then, according to Coulomb's law, for the magnitude $F$ of the projection of the force of interaction on $\mathrm{O}_{1} \mathrm{O}_{2}$, acting on the spheres $S_{1}$ and $S_{2}$, we obtain

$$
F=\frac{1}{4 \pi \varepsilon_{0} R^{2}} \sum_{j=0}^{\infty} \sum_{t=0}^{\infty} \frac{Q_{j}^{\prime} Q_{t}^{\prime \prime}}{\left(1-\delta_{j}^{\prime}-\delta_{t}^{\prime \prime}\right)^{2}}
$$

The potential energy of interaction between the spheres $S_{1}$ and $S_{2}$, according to [16], is

$$
W=\frac{1}{4 \pi \varepsilon_{0} R} \sum_{j=0}^{\infty} \sum_{t=0}^{\infty} \frac{Q_{j}^{\prime} Q_{t}^{\prime \prime}}{1-\delta_{j}^{\prime}-\delta_{t}^{\prime \prime}}
$$

Let us point out that in (7) and (8) we do not take into consideration the interactions between the charges inside the spheres $S_{1}$ and $S_{2}$ as actually the interaction is external-between the charges on the surface of $S_{1}$ with the charges on the surface of $S_{2}$.

Let $M$ be an arbitrary point in the electric field created by charges $Q_{j}^{\prime}$ and $Q_{j}^{\prime \prime} \quad(j=0,1,2, \cdots)$. If $M$ is at distances $a_{j}$ and $b_{j}$ from charges $Q_{j}^{\prime}$ and $Q_{j}^{\prime \prime}$ respectively, then, using the metric relationships in a triangle, we can determine

$$
a_{j}=\sqrt{\frac{\left(a_{0}^{2}-R d_{j}^{\prime}\right)\left(R-d_{j}^{\prime}\right)+b_{0}^{2} d_{j}^{\prime}}{R}}
$$

and

$$
b_{j}=\sqrt{\frac{\left(b_{0}^{2}-R d_{j}^{\prime \prime}\right)\left(R-d_{j}^{\prime \prime}\right)+a_{0}^{2} d_{j}^{\prime \prime}}{R}}
$$

Then based on the principle of linear superposition of states, the potential at point $M$ will be the sum of the potentials of all charges in $M$ [16]. Therefore

$$
V(M)=\frac{1}{4 \pi \varepsilon_{0}} \sum_{j=0}^{\infty}\left(\frac{Q_{j}^{\prime}}{a_{j}}+\frac{Q_{j}^{\prime \prime}}{b_{j}}\right)
$$

It is worthwhile to mention that using Eqs.7-9 one can determine the interaction between two charged spheres for arbitrary small distances between them, which represents a result obtained for the first time.

\section{TOROID MODEL OF NUCLEONS}

Using the results from [14] in [12] we first consider the nucleons as spheres, as they are viewed in the standard model. In this case we show that at distances $\geq 10^{-16} \mathrm{~m}$ we can calculate with good approximation the binding energy and force of interaction between nucleons by modeling protons as point charges. But at distances $<10^{-16} \mathrm{~m}$ the proton should not be considered as a point charge. We also found out that using the standard model the binding energy of triton cannot be obtained. Using this model it is difficult to explain the experimentally obtained magnetic moments of the nuclei relative to the magnetic moments of the comprising nucleons; it is also hard to explain the experimental results for the radii and stability of the nuclei.

For this reason we remodel the nucleons as tori [12]. At that they are rotating with a constant angular velocity around a straight axis $z$, passing through their mass (geometrical) center $O$ and perpendicular to the plane of rotation of their central circle (Figure 1).

After that we study a system of proton and neutron in order to determine the electrostatic interaction between them.

The two tori- of the proton and neutron we denote correspondingly as $T_{p}$ and $T_{n}$ and their centers as $O_{p}$ and $O_{n}$. We also assume that the central circles of $T_{p}$ and $T_{n}$ lie in parallel or coincident planes and rotate in the same or opposite directions with constant angular yelocity around a the straight line $z$ passing through $p$ and $O_{n}$, and perpendicular to the plane of their rotation. Thus if $O_{p} O_{n}=h$, then $h \geq 0$ (Figure 2).

Let us denote by $K_{p}$ and $K_{n}$ the centers of the forming circles of the tori $T_{p}$ and $T_{n}$, and with $R_{p}=O_{p} K_{p}$ and $R_{n}=O_{n} K_{n}$ - the radii of the central circles of $T_{p}$ and $T_{n}$. We assume that $T_{p}$ and $T_{n}$ are at a distance $0<\tau<10^{-15} \mathrm{~m}$ from each other.

According to experimental data the radius of the proton $r_{p}$ is smaller than the radius of the neutron $r_{n}$. Because of that we consider $R_{p}<R_{n}$.

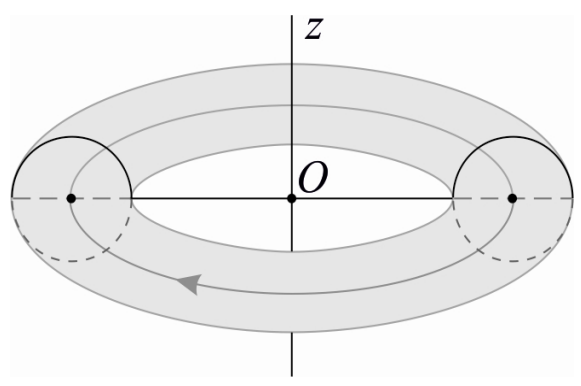

Figure 1. Toroid model of nucleon. 


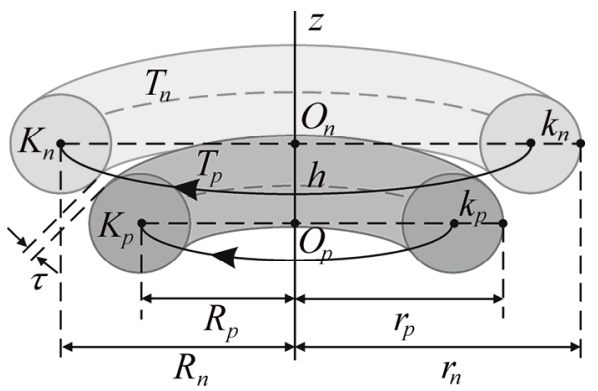

Figure 2. Cross section of a proton-neutron system.

Let $k_{p}$ and $k_{n}$ be the radii of the forming circles $K_{p}$ and $K_{n}$. It is clear that $k_{p}<R_{p}$, and $k_{n}<R_{n}$. Besides that

$$
R_{p}+k_{p}=r_{p} \text { and } R_{n}+k_{n}=r_{n}
$$

where $r_{p}$ and $r_{n}$ are the corresponding radii of the proton and neutron in this configuration. It should be noted that for different nuclei $r_{p}, k_{p}, R_{p}$ and $r_{n}, \mathrm{v}$, $R_{n}$ may have different values.

From geometrical considerations for $O_{p} O_{n}=h$ will be fulfilled the equation

$$
h^{2}=\left(k_{p}+k_{n}+\tau\right)^{2}-\left(R_{n}-R_{p}\right)^{2}
$$

It is clear that $0 \leq h \leq k_{p}+k_{n}+\tau$.

We assume that the volume mass densities of the proton and neutron are equal, i.e. $\rho_{p}=\rho_{n}$. The volumes of the tori $T_{p}$ and $T_{n}$, according to [17], are correspondingly $V_{p}+2 \pi^{2} k_{p}^{2} R_{p}$ and $V_{n}+2 \pi^{2} k_{n}^{2} R_{n}$. Then if $m_{p}$ and $m_{n}$ are the corresponding masses of $T_{p}$ and $T_{n}$, then from $\frac{m_{p}}{V_{p}}=\frac{m_{n}}{V_{n}}$ it follows that

$$
k_{n}=k_{p} \sqrt{\frac{m_{n} R_{p}}{m_{p} R_{n}}}
$$

In (12) we can substitute the experimentally measured masses of the proton and the neutron [18] $m_{p}=1.672621638 \times 10^{-27} \mathrm{~kg}, \quad m_{n}=1.674927212 \times 10^{-27} \mathrm{~kg}$.

Lets denote with $q>0$ the radius of the empty part of the circle with a radius $R_{p}$. Then

$$
q=r_{p}-2 k_{p}
$$

Further let $T_{p}$ and $T_{n}$ from Figure 2 be a protonneutron couple bound in the nucleus of deuterium or tritium. If we denote with $r_{k}$ the radius of the nucleus under consideration, then $r_{n}=r_{k}$.

In order to apply the results from Section 2 for spheres we will remodel the tori.

We will emphasize that the potential energy and the force of interaction between two spheres depend on the distance between image-charges, i.e. from the lengths of the line segments $d_{i, j}(i=1,2 ; j=1,2,3, \cdots)$ from for- mula (3). These lengths in (3) are determined from the squares of the radii $r_{1}$ or $r_{2}$ of the spheres and from the length of their central line $R=r_{1}+r_{2}+\tau$, where $\tau$ is the smallest distance between their surfaces. For the square of the radii of each of the two spheres is fulfilled $r_{i}^{2}=L_{i} / 4 \pi, i=1,2$, where $L_{i}$ is the surface area of the corresponding sphere. Therefore, when we remodel the tori of the proton and the neutron we have to keep both their surface areas $L_{p}$ and $L_{n}$ and the distance $\tau$ between them.

Due to the central symmetry of the charge of the proton we can consider all of its charge $p$ to be concentrated in the geometrical center $O_{p}$ of the torus $T_{p}$.

Therefore we remodel the proton as a sphere $S_{p}$, with radius $\bar{r}_{P}$ and the same centre $O_{p}$ on the straight line $z$ and a surface area equivalent to the torus surface area, i.e. it has the same surface area as the torus $T_{p}$. At that $S_{p}$ is charged with a charge $p$ that is centrally symmetrical and can be redistributed.

The surface area $L_{p}$ of a torus $T_{p}$ according to [17] is

$$
L_{p}=4 \pi^{2} k_{p} R_{p}
$$

Then, as the surface areas of the torus $T_{p}$ and the sphere $S_{p}$ are equal, then from (14) it follows that the radius of $S_{p}$ is

$$
\bar{r}_{P}=\sqrt{\pi k_{p} R_{p}}
$$

We remodel the neutron $T_{n}$ with a torus $T_{N}$ with an equivalent surface area. At that $T_{N}$ has the same center $O_{n}$ on $z$ and its surface is at the same distance $\tau$ from the surface of $S_{p}$ as in the case of $T_{n}$ and $T_{p}$ (Figure 3).

Let $S_{N}$ be a sphere. Its central circle, is forming for the torus $T_{N}$. We denote the centre of $S_{N}$ with $K_{N}$, at that $O_{n} K_{N}=R_{N}$ and with $\bar{r}_{N}$-the radius of $S_{N}$. Thus if $O_{p} K_{N}=\bar{R}$ then $\bar{R}=\bar{r}_{P}+\bar{r}_{N}+\tau$ and from $O_{p} O_{n}=h$ it follows that $R_{N}{ }^{2}=\bar{R}^{2}-h^{2}$, i.e.

$$
R_{N}=\sqrt{\left(\bar{r}_{P}+\bar{r}_{N}+\tau\right)^{2}-h^{2}}
$$

It is clear that $\max R_{N}=\bar{r}_{p}+\bar{r}_{N}+\tau$ and $\min R_{N}=\bar{r}_{N}$.

From the equality of the surface areas $L_{N}$ and $L_{n}$ of the tori $T_{N}$ and $T_{n}$, it follows that

$$
\bar{r}_{N}=k_{n} \frac{R_{n}}{R_{N}}
$$

For the number $l$ of spheres $S_{N}$ which have total surface area equal to the surface area of the torus $T_{N}$ is fulfilled $l .4 \pi \bar{r}_{N}^{2}=4 \pi^{2} R_{N} \bar{r}_{N}$. Therefore

$$
l=\pi \frac{R_{N}}{\bar{r}_{N}}
$$

We assume that the centre $O_{p}$ is motionless relative to the inertial reference system $J$. We introduce a solid non-inertial reference system $G$ that rotates with the con- 
stant angular velocity $\omega$ of rotation of $T_{N}$ relative to $J$. At that, the point $O_{p}$ is the centre of the coordinate system $O_{p} x y z$, stationary connected with $G$ and relative to which the spheres $S_{P}$ and $S_{N}$ are motionless to each other (Figure 3).

Then for the experimentally obtained value of $r_{k}=r_{n}$, giving values to $q$, from Eqs.10-13 we calculate $R_{p}$, $k_{p}, r_{p}, R_{n}$ and $k_{n}$. With those and formula (15) we obtain the radius $\bar{r}_{P}=\sqrt{\pi k_{p} R_{p}}$ of the surface-equivalent sphere $S_{P}$. Varying $0<\tau<10^{-15} \mathrm{~m}$ and $0 \leq h \leq k_{p}+k_{n}+\tau$, from formulas (16) and (17) wecalculate the radius $\bar{r}_{N}$ of the forming sphere $S_{N}$.

Thus at $\bar{R}=\bar{r}_{P}+\bar{r}_{N}+\tau$, through formulas (7) and (8), we find the binding energy $W\left(S_{P}, S_{N}\right)$ and force of interaction $F\left(S_{P}, S_{N}\right)$. According to (18), the binding energy $W\left(T_{p}, T_{n}\right)$ and force of interaction $F\left(T_{p}, T_{n}\right)$ between a the proton and the neutron will be

$$
\begin{aligned}
& W\left(T_{p}, T_{n}\right)=\pi \frac{R_{N}}{r_{N}} W\left(S_{P}, S_{N}\right) \\
& F\left(T_{p}, T_{n}\right)=\pi \frac{R_{N}}{r_{N}} F\left(S_{P}, S_{N}\right)
\end{aligned}
$$

The Eqs.19 are valid because the forming spheres of the torus $T_{N}$ are situated symmetrically relative to the centre of the sphere $S_{P}$.

Moving on we will structure the nuclei of deuterium and tritium. The positioning of the nucleons in them must correspond to the principle of minimum potential energy. Due to the mass defect in the nucleus, the potential energy of the interaction in the atomic nuclei is calculated by the following formula [2]:

$$
W_{K}=\left(N_{p} m_{p}+N_{n} m_{n}-m_{K}\right) c^{2}
$$

Here $N_{p}$ is the number of the protons, $N_{n}-$ number of the neutrons; $m_{p}=1.672621638 \times 10^{-27} \mathrm{~kg}$ and $m_{n}=1.674927212 \times 10^{-27} \mathrm{~kg}$ are the masses of the proton and of the neutron, respectively [18] and $m_{K}$ is the mass of the considered nucleus; $c=2.99792458 \times 10^{8}$ $\mathrm{m} \cdot \mathrm{s}^{-1}$ is the velocity of the light in flat vacuum [18]. We will find the values of $W_{K}$ according to our method with formula (8), comparing them with the values obtained through formula (20).

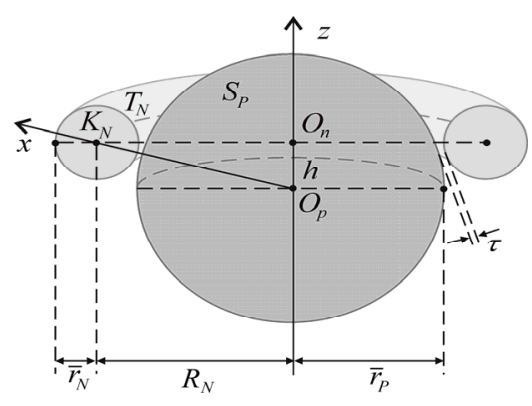

Figure 3. Cross section of reduced model of the proton-neutron system.
Let us consider the proton in a unbound state as a torus $T_{p}^{0}$. Its radius according to [19] is $r_{p}=0.84184 \times 10^{-15} \mathrm{~m}$. If $K_{p}^{0}$ is the center of the forming circle of $T_{p}^{0}$, then we denote as $k_{p}^{0}$ the radius of this circle and with $R_{p}^{0}=O K_{p}^{0}$.

Using formulas (10) and (13) for different values of the radius of the circle of the empty part $q$ we find $k_{p}^{0}$, $R_{p}^{0}, V_{p}^{0}$ and $\rho_{p}^{0}$. We also find the volume $V_{n}^{0}$ of the neutron in a free state since we know its mass $m_{n}^{0}$ and we have assumed that $\rho_{p}^{0}=\rho_{n}^{0}$. All the data are given in Table 1.

In Table 1 we have denoted with $k_{p}$ the radius of the forming circle of the torus of the proton and with $R_{p}$ the radius of the central circle of the torus of the proton.

Due to the mass defect in atomic nuclei the volumes of the nucleons within them change. We will assume however that the volume mass densities of the proton and neutron do not change, i.e. $\rho_{p}^{0}=\rho_{n}^{0}=\rho=$ const .

\section{MODEL OF THE DEUTERON}

We model the nucleus of deuterium as two concentric toroids inlaid one in the other. The internal torus $T_{p}$ corresponds to the proton and the external one $T_{n}$ corresponds to the neutron with $T_{p}$ and $T_{n}$ being at a distance $0<\tau<10^{-15} \mathrm{~m}$. This configuration provides symmetry relative to the center of masses $O$ of the deuteron placed in its geometrical center (Figure 4). From that follows the stability of the deuteron $T=\infty$.

We assume that both tori spin in the same direction with a constant angular velocity $\omega$ around the line $z$ going through their common center $O=O_{p}=O_{n}$ and perpendicular to their plane of rotation.

Table 1. Size, volume and mass density of the proton and neutron.

\begin{tabular}{cccccc}
\hline $\begin{array}{c}q \\
\mathrm{~m}\end{array}$ & $\begin{array}{c}k_{p} \\
\mathrm{~m} \times 10^{-15}\end{array}$ & $\begin{array}{c}R_{p} \\
\mathrm{~m} \times 10^{-15}\end{array}$ & $\begin{array}{c}V_{p}^{0} \\
\mathrm{~m}^{3} \times 10^{-45}\end{array}$ & $\begin{array}{c}V_{n}^{0} \\
\mathrm{~m}^{3} \times 10^{-45}\end{array}$ & $\begin{array}{c}\rho_{p}^{0}=\rho_{n}^{0} \\
\mathrm{~kg} \cdot \mathrm{m}^{-3} \times 10^{18}\end{array}$ \\
\hline $0.3 r_{p}$ & 0.29464 & 0.54720 & 0.93771 & 0.939001 & 1.78373 \\
$0.4 r_{p}$ & 0.25255 & 0.58929 & 0.74192 & 0.742946 & 2.25444 \\
$0.5 r_{p}$ & 0.21046 & 0.63138 & 0.55203 & 0.552787 & 3.02997 \\
$0.6 r_{p}$ & 0.16837 & 0.67347 & 0.37685 & 0.377369 & 4.43843 \\
$0.7 r_{p}$ & 0.12628 & 0.71556 & 0.22523 & 0.225537 & 7.42639 \\
\hline
\end{tabular}

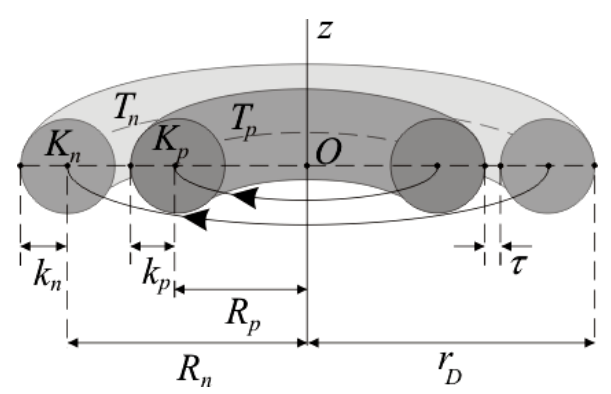

Figure 4. Cross-section of the model of the deuteron. 
From this structure follows that the spin $s_{D}$ of the deuteron will be a sum of the spins $s_{p}=s_{n}=1 / 2$ of the proton and neutron, i.e. the spin of the deuteron is $s_{D}=1$, which has been experimentally obtained.

The nucleons, binding to each other within the deuteron almost double their total mass relative to their separate masses and as a consequence the centrifugal force increases. From this follows that the radius of the deuteron is larger than the radii of the nucleons and as has been experimentally determined it is $r_{D}=2.14 \times 10^{-15} \mathrm{~m}$ [18].

Let us assume that the charge of each of the nucleons is distributed parallel along a circle with a center $O$. Then a circular current appears and the magnetic moments of the nucleons are proportional to the magnitude of the charges and their angular velocity but also to the square of their distance from the center of rotation. The inlaying of the proton within the neutron increases the radius of the neutron more relative to the increased radius of the proton. This explains why the sum of the magnetic moments of the proton $\mu_{p}=1.4106 \times 10^{-26} \mathrm{JT}^{-1}$ and neutron $\mu_{n}=-0.9662 \times 10^{-26} \mathrm{JT}^{-1}$ is larger than the magnetic moment of the deuteron

$$
\mu_{D}=0.4331 \times 10^{-26} \mathrm{JT}^{-1}[18] \text {. }
$$

Let $K_{p}$ and $K_{n}$ be the centers of the forming circles of $T_{p}$ and $T_{n}$ and let the radii of those circles be $k_{p}$ and $k_{n}$ correspondingly. We denote as $R_{p}=O K_{p}$ and $R_{n}=O K_{n}$ the radii of the central circles of the tori $T_{p}$ and $T_{n}$ (Figure 4).

In this case $O_{p}=O_{n}=O$ and $h=O_{p} O_{n}=0$, also $R_{n}=r_{D}-k_{n}$ and $R_{p}=r_{D}-2 k_{n}-k_{p}-\tau$. Based on the method we described in Section 3 using mass volume density $\rho$ of the nucleons from Table 1 we find $k_{n}$, $R_{n}, k_{p}$ and $R_{p}$. In formula (12) we consider the mass defect, proportionally for the masses of the proton and neutron. The experimentally obtained mass of the deuteron is $m_{D}=3.3436 \times 10^{-27} \mathrm{~kg}$ [18]. Then, according to formula (20), the binding energy of the deuteron is $W_{D}=-3.5642 \times 10^{-13} \mathrm{~J}$.

This value we confirm at different values of the distance $\tau$ between the tori (Table 2) for $W\left(T_{p}, T_{n}\right)$ with the corresponding formula from (19). From (19) we also obtain the force of interaction $F\left(T_{p}, T_{n}\right)$ for the deuteron.

In Table 2 we have denoted with $k_{n}$ and $k_{p}$ respectively the radius of the forming circle of the torus of the neutron and the proton, with $R_{n}$ and $R_{p}$ respecttively the radius of the central circle of the torus of the neutron and the proton and with $F_{D}$ the force of interaction between the nucleons in the deuteron.

\section{MODEL OF THE TRITON}

The triton is obtained structurally from the deuteron by adding one more neutron. The second neutron tries to take over the place of the first one.

The neutron, although it can be assumed electrically neutral, i.e. with a common charge $n=0$, has an internal electric structure, its negative charge, as opposed to the positive one is distributed primarily at its surface $[20,21]$.

Then because of the repulsion between the tori $T_{n}^{1}$ and $T_{n}^{2}$ the central circles of the nucleons will be situated symmetrically in two planes parallel to the plane in which the central circle of proton torus $T_{p}$ is situated. At that $T_{n}^{1}$ and $T_{n}^{2}$ will be at the same distance $0<\tau<10^{-15} \mathrm{~m}$ from $T_{p}$. This configuration provides symmetry relative to the center of masses (geometrical center) $O$ of the triton (Figure 5). From this follows the relative stability of the triton $T=12.262 y$.

Let us denote as $O_{n}^{i}$ the centers of the tori $T_{n}^{i}$ $(i=1,2)$ and as $O_{p}$ - the center of $T_{p}$. Then the center $O=O_{p}$ and the points $O_{n}^{i}$ are on the same line $Z$, perpendicular to their plane of rotation.

The tori $T_{p}$ and $T_{n}^{1}$ rotate around $z$ with a constant angular velocity $\omega$ in the same direction (e.g. clockwise) and $T_{n}^{2}$ will rotate with the same velocity in the opposite direction (counterclockwise), i.e. with a velocity $-\omega$. Thus we obtain that the spin of the triton is $s_{T}=1 / 2$ which is experimentally confirmed.

Table 2. Size of the nucleons and force of interaction in the deuteron.

\begin{tabular}{ccccccc}
\hline $\begin{array}{c}\tau \\
\mathrm{kg} \cdot \mathrm{m}^{-3} \times 10^{18}\end{array}$ & $\begin{array}{c}k_{n} \times 10^{-17} \\
\mathrm{~m} \times 10^{-15}\end{array}$ & $\begin{array}{c}R_{n} \\
\mathrm{~m} \times 10^{-15}\end{array}$ & $\begin{array}{c}k_{p} \\
\mathrm{~m} \times 10^{-15}\end{array}$ & $\begin{array}{c}R_{p} \\
\mathrm{~m} \times 10^{-15}\end{array}$ & $\begin{array}{c}F_{D} \\
\mathrm{~N}\end{array}$ \\
\hline 1.78373 & 2.30200 & 0.15489 & 1.9851 & 0.17047 & 1.6367 & -1962 \\
2.25444 & 2.28778 & 0.13717 & 2.0028 & 0.14906 & 1.6937 & -2045 \\
3.02997 & 2.27329 & 0.11775 & 2.0223 & 0.12629 & 1.7555 & -2147 \\
4.43843 & 2.25592 & 0.096787 & 2.0432 & 0.10244 & 1.8214 & -2284 \\
7.42639 & 2.23334 & 0.074418 & 2.0656 & 0.077722 & 1.8911 & -2479 \\
\hline
\end{tabular}

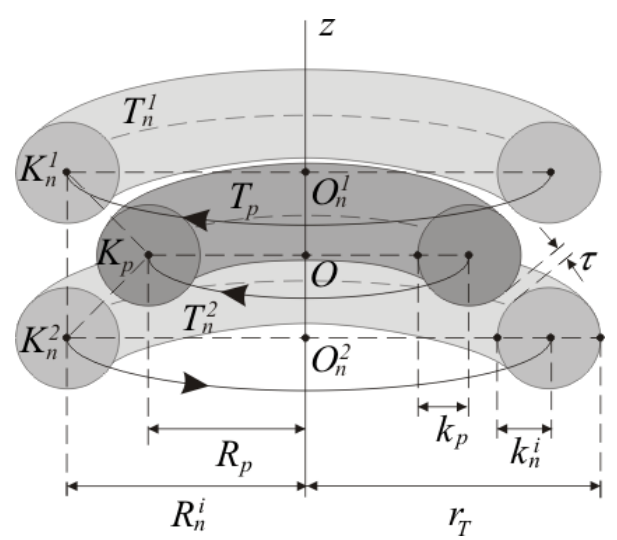

Figure 5. Cross-section of the model of the triton. 
The neutron that rotates in an opposite direction will decrease the centrifugal force caused by the nucleons rotating in the same direction. Therefore the radius of the triton $r_{T}<r_{D}$. According to experiments $r_{T}=1.6 \times 10^{-15}$ $\mathrm{m}[22]$.

The magnetic moments caused by the redistributed charges in the neutrons cancel each other out. Thus the magnetic moment of the triton is caused only by the charge of the proton. As a consequence of the increased radius of the proton relative to the free state the magnetic moment of the triton $\mu_{T}=1.5046 \times 10^{-26} \mathrm{JT}^{-1}$ is larger than the magnetic moment of the proton $\mu_{p}=1.4106 \times 10^{-26}$ $\mathrm{JT}^{-1}$, as shown by experimental data [18].

Let $K_{p}$ and $K_{n}^{i}$ be the centers of the forming circles respectively of $T_{p}$ and $T_{n}^{i}$ with corresponding radii $k_{p}$ and $k_{n}^{i}(i=1,2)$. We denote $O K_{p}=R_{p}$ and for $i=1,2, O_{n}^{i} K_{n}^{i}=R_{n}^{i}$.

In this case $h=O O_{n}^{i}$ and if $r_{T}$ is the radius of the triton, then $R_{n}^{i}=r_{T}-k_{n}^{i}$.

Due to the central symmetry of the charge of the proton we can assume that all of its charge $p>0$ is concentrated in the geometrical center $O$. Because of that we model the proton as a sphere $S_{P}$ with a center $O$ and radius $\bar{r}_{p}$, equivalent in surface are to $T_{p}$. At that $S_{p}$ is charged with a charge $p$, which is centrally symmetrical and can be redistributed.

With $T_{N}^{i} \quad(i=1,2)$ we denote the tori that are equivalent in surface to $T_{n}^{i}$ with centers $O_{n}^{i}$ and centers of the forming circles $K_{N}^{i}$ (Figure 6). At that $S_{P}$ and $T_{N}^{i}$ have the same distance $\tau$ between them as $T_{p}$ and $T_{n}^{i}$. With $S_{N}^{i} \quad(i=1,2)$ we denoted the forming sphere of $T_{N}^{i}$.

We assume the point $O$ to be stationary relative to the inertial reference frame $J$. We introduce two solid noninertial reference systems $G_{1}$ and $G_{2}$, that rotate with the constant angular velocities $\omega$ and $-\omega$ respectively of $T_{N}^{1}$ and $T_{N}^{2}$ relative to $J$.

The point $O$ is a center of the coordinate system $O x_{i} y_{i} z$ stationary connected with the reference system $G_{i} \quad(i=1,2)$ relative to which the spheres $S_{P}$ and $S_{N}^{i}$ are stationary to each other (Figure 6).

The two tori $T_{N}^{1}$ and $T_{N}^{2}$ are symmetrical relative to the sphere $S_{P}$. Then it is enough to study the electrostatic interaction only between one of them and $S_{P}$. We assume with some approximation that between $T_{N}^{1}$ and $T_{N}^{2}$ there is no electrostatic interaction since their total charges are zero. Besides that, to simplify the calculations, we assume that the distance between the surfaces of each of the tori is the same and equal to $\tau$. Then the distance $h=k_{n}+\tau / 2$.

Since $r_{n}=r_{T}$, using the model we revealed in Section 3 , with the volume mass density $\rho$ of the nucleons from Table 1 we find $k_{n}^{i}, R_{n}^{i}$ and $k_{p}, R_{p}$. In formula (12) we consider the mass defect proportionally for the masses of the proton and two neutrons. The experimenttally obtained mass of the triton is $m_{T}=5.00735588 \times 10^{-27}$ $\mathrm{kg}$ [18].

Then, according to formula (20) the binding energy of the triton is $W_{T}=-13.5895 \times 10^{-13} \mathrm{~J}$. This value we confirm for different values of the distance $\tau$ between the tori (Table 3) for $W_{T}=2 W\left(T_{p}, T_{n}^{i}\right), \quad i=1$ or $i=2$ using the corresponding formula from (19). From (19) we also obtain the force of interaction for the triton $F_{T}=2 F\left(T_{p}, T_{n}^{i}\right)$ for $i=1$ or $i=2$.

In Table 3 we have denoted with $k_{n}$ and $k_{p}$ respectively the radius of the forming circle of the torus of the neutron and the proton, with $R_{n}$ and $R_{p}$ respectively the radius of the central circle of the torus of the neutron and the proton and with $F_{T}$ the force of interaction between the nucleons in the triton.

We should note that by determining the value of $W_{T}$, we can also vary $h$, where in this case is fulfilled $k_{n}<h \leq k_{p}+k_{n}+\tau$.

\section{DISCUSSION}

In [12] considering the nucleons as tori we theoreticcally determine the potential energy and the force of interaction in the systems: proton-neutron, proton-proton and proton-neutron-proton, which we derive using experimentally obtained results for the radii and the masses of the nucleons in unbound condition.

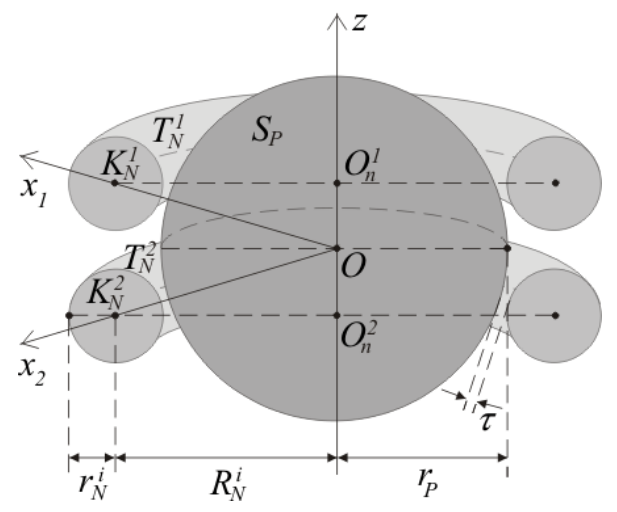

Figure 6. Cross-section of the reduced model of the triton.

Table 3. Size of the nucleons and force of interaction in the triton.

\begin{tabular}{ccccccc}
\hline$\rho$ & $\tau$ & $k_{n}$ & $R_{n}$ & $k_{p}$ & $R_{p}$ & $F_{T}$ \\
$\mathrm{~kg} \cdot \mathrm{m}^{-3} \times 10^{18}$ & $\mathrm{~m} \times 10^{-17}$ & $\mathrm{~m} \times 10^{-15}$ & $\mathrm{~m} \times 10^{-15}$ & $\mathrm{~m} \times 10^{-15}$ & $\mathrm{~m} \times 10^{-15}$ & $\mathrm{~N}$ \\
\hline 1.78373 & 1.17113 & 0.18353 & 1.4165 & 0.21248 & 1.0554 & -9979 \\
2.25444 & 1.16356 & 0.16203 & 1.4380 & 0.18322 & 1.1230 & -10393 \\
3.02997 & 1.16033 & 0.13864 & 1.4614 & 0.15324 & 1.1944 & -11045 \\
4.43843 & 1.15927 & 0.11358 & 1.4864 & 0.12284 & 1.2690 & -11649 \\
7.42639 & 1.15838 & 0.08703 & 1.5130 & 0.092201 & 1.3462 & -12933 \\
\hline
\end{tabular}


Using our method, we have shown that the electromagnetic forces for the proton-neutron pair are quite strong (in the order of the nuclear forces) an short-ranged. This suggests that the binding energy of the nucleons have electromagnetic nature. We can also explain other basic experimental data like stability, radius, magnetic moment and spin of the nuclei.

In this paper, we concretize the general results obtained in [12] for the nuclei of deuterium and tritium.

\section{CONCLUSIONS}

Nuclear physics bases its knowledge on experiments and has numerous different contradicting models. Considering nucleons as tori and modeling the deuteron and triton, we obtain and explain their basic experimentally obtained characteristics and also obtain new characteristics of these nuclei.

Our model can also be applied for more complicated atomic nuclei. Based on particular charge and current configurations to it can be considered the interaction betweennucleons in electrodynamic aspect; to be find analytical expressions for the magnetic moments, to determine the angular velocity of nucleons, the linear velocity at particular points on their surface, etc.; to explain excited states of the nuclei; to be find out the potential of the electromagnetic field generated by atomic nuclei and to calculate their quadrupole moments for the deuteron and the other nuclei.

\section{ACKNOWLEDGEMENTS}

The authors express their gratitude to the computer specialist Stefan Bozhkov, who with the help of Wolfram Mathematica 7.0 performs the calculations in this article. The results of the present studies are published with the financial support of the Fund for Scientific Research with the Ministry of Education and Science under contract DTC No. $02 / 35$.

\section{REFERENCES}

[1] Cook, N. (2006) Models of the atomic nucleus. Springer, Berlin.

[2] Krane, K. (1999) Introductory nuclear physics. WileyVCH, New York.

[3] Martin, B. (2008) Nuclear and particle physics. 3rd Edition, Wiley, New York.

[4] Schopper, H., Altarelli, G., Grünewald, M. and Inoue, K.
(2008) Elementary particles. Springer, Berlin.

[5] Standard model. http://en.wikipedia.org/wiki/Standard_Model

[6] University of Tennessee. Standard model. http://electron6.phys.utk.edu/phys $250 /$ modules/module $\%$ 206/standard model.htm

[7] Fehling, D., The standard model of particle physics: A lunchbox's guide. http://www.pha.jhu.edu/ dfehling/

[8] Bergman, D. (2000) The real proton. Foundations of Science, 3,4 .

[9] Toroidal ring model. http://www.enotes.com/topic/Toroidal ring model

[10] Toroidal ring model. http://en.wikipedia.org/wiki/Toroidal_ring_model

[11] Twain, M. (1995) The undiscovered physics. http://groupkos.com/mtwain/TheProton.pdf.

[12] Kolikov, K., Ivanov, D. and Krustev, G. (2012) Electromagnetic nature of the nuclear forces and a toroid model of nucleons in atomic nuclei. Natural Science, 4, 47-56. doi:10.4236/ns.2012.41008

[13] Blokhintsev, D. (1983) Basics of quantum mechanics. Nauka (in Russian).

[14] Kolikov, K., Ivanov, D., Krustev, G., Epitropov Y. and Bozhkov, S. (2012) Electrostatic interaction between two conducting spheres. Journal of Electrostatics, 70, 91-96. doi:10.1016/j.elstat.2011.10.008

[15] Feynman, R. (1964) The feynman lectures on physics: Exercises. Addison Wesley Publishing Co., New York.

[16] Halliday, D., Resnick, R. and Walker, J. (2011) Fundamentals of physics. 9th Edition, John Wiley \& Sons, Inc., New York.

[17] Gellert, W., Kästner, H. and Neuber, S. (1983) Mathematical encyclopedic dictionary. Science and Art, Sofia, 585 (in Bulgarian).

[18] Mohr, P., Taylor, B. and Newell, D. (2008) CODATA recommended values of the fundamental physical constants: 2006. http://arxiv.org/abs/0801.0028.

[19] Sick, I. (2003) On the rms-radius of the proton. Physics Letters B, 576, 62-67. doi:10.1016/j.physletb.2003.09.092

[20] Hofstadter, R. (1956) Electron scattering and nuclear structure. Reviews of Modern Physics, 28, 213. doi:10.1103/RevModPhys.28.214

[21] Burcham, W.E. (1963) Nuclear physics. McGraw-Hill Book Co., Inc., New York.

[22] Kirscher, J., Griesshammer, H., Shukla, D. and Hofmann, H. (2010) Universal correlations in pion-less EFT with the resonating group method: Three and four nucleons. European Physical Journal A, 44, 239-256. 